\title{
Thermodynamics of Helium-4 Dimerization and Trimerization
}

\author{
Arthur M. Halpern \\ Department of Chemistry and Physics, Indiana State University, Terre Haute, IN 47809, United \\ States \\ Email: arthur.halpern@indstate.edu
}

\section{SUPPORTING INFORMATION}

\section{Derivation of $r=1 / Z$ (equation 10 of the article)}

Suppose $n_{\text {id }}$ moles of an ideal gas are placed in a container of volume $V_{\text {id }}$ and kept at a constant temperature $T$ and pressure $P$. If, however, the gas is now considered to be nonideal, association must be taken into account. After equilibrium is reached, there are $n_{1}$ moles of the original, monomeric species, $n_{2}$ moles of dimer, $n_{3}$ moles of trimer, etc. The volume now becomes $V_{\text {eq. }}$. A stoichiometric accounting of the mixture gives

$$
n_{\text {id }}=n_{1}+2 n_{2}+3 n_{3}+\mathrm{L}=\sum_{i} i n_{i},
$$

and total number of moles (of the real gas) in the container at equilibrium is

$$
n_{\mathrm{eq}}=n_{1}+n_{2}+n_{3}+\mathrm{K}=\sum_{i} n_{i} .
$$

We now define $r$ as the ratio of these mole numbers,

$$
r \equiv \frac{n_{\mathrm{id}}}{n_{\mathrm{eq}}}=\frac{\sum_{i} i n_{i}}{\sum_{i} n_{i}} .
$$

Since $n_{\mathrm{eq}}<n_{\mathrm{id}}$ it follows that $r>1$.

The compression factor $Z$ is defined as the volume occupied by the (real) gas under equilibrium conditions, $V_{\text {eq }}$, divided by the volume the gas would occupy if it were ideal and at the same temperature and pressure. Thus,

$$
Z=\frac{V_{\text {eq }}}{V_{\text {id }}} .
$$

Applying Avogadro's law we have $Z=n_{\mathrm{eq}} / n_{\mathrm{id}}$. Since $Z$ is also expressed as the quotient $P V_{\mathrm{m}} / R T$ (obtained from the equation of state), where $V_{\mathrm{m}}$ is the molar volume (at equilibrium), we can write

$$
r_{\mathrm{EOS}}=\frac{1}{Z}=\frac{R T}{P V_{\mathrm{m}}}
$$

which corresponds to equation 10 of the article. 


\section{Derivation of eq 12 of the article}

Equation 11 of the article reads

$$
\left(r_{\mathrm{EOS}}-1\right)=x_{\mathrm{D}}+2 x_{T}=K_{\mathrm{D}}^{\mathrm{o}} P x_{\mathrm{M}}^{2}+2 K_{\mathrm{T}}^{\mathrm{o}} P^{2} x_{\mathrm{M}}^{3},
$$

which is used to express $\left(2-r_{\mathrm{EOS}}\right)^{2}$

$$
\left(2-r_{\mathrm{EOS}}\right)^{2}=\left(1-x_{\mathrm{D}}-2 x_{\mathrm{T}}\right)^{2}=1-2 x_{\mathrm{D}}-4 x_{\mathrm{T}}+x_{\mathrm{D}}^{2}+4 x_{\mathrm{T}}^{2}+4 x_{\mathrm{D}} x_{\mathrm{T}}
$$

Replacing $x_{\mathrm{D}}$ and $x_{\mathrm{T}}$ with

$$
x_{\mathrm{D}}=K_{\mathrm{D}}^{\mathrm{o}} P x_{\mathrm{M}}^{2} \text { and } x_{\mathrm{T}}=K_{\mathrm{T}}^{\mathrm{o}} P^{2} x_{\mathrm{M}}^{3}
$$

respectively, and using eq SI-6, it follows that

$$
\frac{\left(r_{\mathrm{EOS}}-1\right)}{\left(2-r_{\mathrm{EOS}}\right)^{2}}=\frac{K_{\mathrm{D}}^{\mathrm{o}} P x_{\mathrm{M}}^{2}+2 K_{\mathrm{T}}^{\mathrm{o}} P^{2} x_{\mathrm{M}}^{3}}{1-2 K_{\mathrm{D}}^{\mathrm{o}} P x_{\mathrm{M}}^{2}-4 K_{\mathrm{T}}^{\mathrm{o}} P^{2} x_{\mathrm{M}}^{3}+K_{\mathrm{D}}^{\mathrm{o} 2} P^{2} x_{\mathrm{M}}^{4}+4 K_{\mathrm{D}}^{\mathrm{o}} K_{\mathrm{T}}^{\mathrm{o}} P^{3} x_{\mathrm{M}}^{5}+4 K_{\mathrm{T}}^{\mathrm{o} 2} P^{4} x_{\mathrm{M}}^{6}}
$$

At sufficiently low pressure, where $x_{\mathrm{M}} \cong 1$, the denominator $\cong 1$, and thus

$$
\frac{\left(r_{\mathrm{EOS}}-1\right)}{\left(2-r_{\mathrm{EOS}}\right)^{2}} \cong K_{\mathrm{D}}^{\mathrm{o}} P+2 K_{\mathrm{T}}^{\mathrm{o}} P^{2}
$$

A second order regression is performed on eq SI-10 to obtain initial values of $K_{\mathrm{D}}^{\mathrm{o}}$ and $K_{\mathrm{T}}^{\mathrm{o}}$ for use in the multiple regression of SI-6 (eq 11 of the article) to obtain refined values and their standard uncertainties. 
III. Table SI-1. Standard thermodynamic properties of the helium dimer equilibrium between 3.0 and 10.0 K. Rows $A$ and $B$ contain results obtained from the EOS and statistical thermodynamic calculations, respectively.

\begin{tabular}{|c|c|c|c|c|c|}
\hline$T / \mathrm{K}$ & Note & $K_{\mathrm{D}}^{\mathrm{o}}$ & $\Delta H_{\mathrm{D}}^{\mathrm{o}}$ & $\Delta G_{\mathrm{D}}^{\mathrm{o}}$ & $\Delta S_{\mathrm{D}}^{\mathrm{o}}$ \\
\hline \multirow[t]{2}{*}{3.0} & $\mathrm{~A}$ & 0.48317 & -54.53 & 18.14 & -24.22 \\
\hline & B & 0.87313 & -62.36 & 3.39 & -21.91 \\
\hline \multirow[t]{2}{*}{3.5} & $\mathrm{~A}$ & 0.34464 & -65.58 & 31.00 & -27.60 \\
\hline & B & .59390 & -72.75 & 15.17 & -25.12 \\
\hline \multirow[t]{2}{*}{4.0} & $\mathrm{~A}$ & .25615 & -75.60 & 45.30 & -30.22 \\
\hline & B & 0.42534 & -83.14 & 28.43 & -27.89 \\
\hline \multirow[t]{2}{*}{4.5} & $\mathrm{~A}$ & 0.19651 & -85.84 & 60.88 & -32.60 \\
\hline & B & 0.31685 & -9354 & 43.01 & -30.34 \\
\hline \multirow[t]{2}{*}{5.0} & $\mathrm{~A}$ & 0.15456 & -96.38 & 77.62 & -34.80 \\
\hline & B & 0.24348 & -103.93 & 58.73 & -32.53 \\
\hline \multirow[t]{2}{*}{5.5} & A & 0.12403 & -107.28 & 95.45 & -36.86 \\
\hline & B & 0.19186 & -114.32 & 75.50 & -34.51 \\
\hline \multirow[t]{2}{*}{6.0} & A & 0.10116 & -118.59 & 114.29 & -38.81 \\
\hline & B & 0.15435 & -124.72 & 93.22 & -36.32 \\
\hline \multirow[t]{2}{*}{6.5} & $\mathrm{~A}$ & 0.083623 & -130.33 & 134.11 & -40.68 \\
\hline & B & 0.12636 & -135.11 & 111.80 & -37.53 \\
\hline \multirow[t]{2}{*}{7.0} & A & 0.069912 & -142.57 & 154.85 & -42.49 \\
\hline & $\mathrm{B}$ & 0.10499 & -145.50 & 131.19 & -39.53 \\
\hline \multirow[t]{2}{*}{7.5} & A & 0.059008 & -155.33 & 176.48 & -44.24 \\
\hline & $\mathrm{B}$ & 0.088354 & -155.90 & 151.31 & -40.96 \\
\hline \multirow[t]{2}{*}{8.0} & $\mathrm{~A}$ & 0.050207 & -168.67 & 198.99 & -45.96 \\
\hline & B & 0.075189 & -166.29 & 172.13 & -42.30 \\
\hline \multirow[t]{2}{*}{8.5} & A & 0.043016 & -182.64 & 222.35 & -47.65 \\
\hline & $\mathrm{B}$ & 0.064615 & -176.68 & 193.60 & -43.56 \\
\hline \multirow[t]{2}{*}{9.0} & A & 0.037072 & -197.31 & 246.56 & -49.32 \\
\hline & $\mathrm{B}$ & 0.056011 & -187.08 & 215.68 & -44.75 \\
\hline \multirow[t]{2}{*}{9.5} & A & 0.032112 & -212.20 & 271.60 & -50.93 \\
\hline & $\mathrm{B}$ & 0.048930 & -197.47 & 238.34 & -45.87 \\
\hline \multirow[t]{2}{*}{10.0} & A & 0.027936 & -226.67 & 297.48 & -52.42 \\
\hline & B & 0.043041 & -207.86 & 261.55 & -46.94 \\
\hline
\end{tabular}


IV. Table SI-2. Standard thermodynamic properties of the helium trimer equilibrium between 3.0 and 10.0 K. Rows $A$ and $B$ contain results obtained from the EOS and statistical thermodynamic calculations, respectively.

\begin{tabular}{|c|c|c|c|c|c|}
\hline$T / \mathrm{K}$ & Note & $K_{\mathrm{T}}^{\circ}$ & $\Delta H_{\mathrm{T}}^{\circ}$ & $\Delta G_{\mathrm{T}}^{\mathrm{o}}$ & $\Delta S_{\mathrm{T}}^{\circ}$ \\
\hline \multirow[t]{2}{*}{3.0} & A & 0.4876 & -111.0 & 17.92 & -42.97 \\
\hline & $\mathrm{B}$ & 0.5005 & -125 & 17.3 & -47.7 \\
\hline \multirow[t]{2}{*}{3.5} & A & 0.2449 & -133.8 & 40.94 & -49.93 \\
\hline & B & 0.2312 & -149 & 42.6 & -54.8 \\
\hline \multirow[t]{2}{*}{4.0} & $\mathrm{~A}$ & 0.1335 & -154.6 & 66.96 & -55.40 \\
\hline & B & 0.1184 & -170 & 71.0 & -60.1 \\
\hline \multirow[t]{2}{*}{4.5} & A & 0.07760 & -176.1 & 95.64 & -60.38 \\
\hline & B & 0.06567 & -190 & 102 & -64.9 \\
\hline \multirow[t]{2}{*}{5.0} & A & 0.04739 & -198.3 & 126.77 & -65.01 \\
\hline & $\mathrm{B}$ & 0.03875 & -211 & 135 & -69.1 \\
\hline \multirow[t]{2}{*}{5.5} & A & 0.03010 & -221.5 & 160.20 & -69.41 \\
\hline & B & 0.02405 & -231 & 170 & -73.0 \\
\hline \multirow[t]{2}{*}{6.0} & A & 0.01974 & -246.0 & 195.81 & -73.63 \\
\hline & $\mathrm{B}$ & 0.01556 & -251 & 208 & -76.6 \\
\hline \multirow[t]{2}{*}{6.5} & A & 0.01329 & -271.9 & 233.49 & -77.75 \\
\hline & $\mathrm{B}$ & 0.01042 & -272 & 247 & -80.0 \\
\hline \multirow[t]{2}{*}{7.0} & A & 0.009142 & -299.5 & 273.25 & -81.82 \\
\hline & $\mathrm{B}$ & 0.007192 & -293 & 287 & -82.9 \\
\hline \multirow[t]{2}{*}{7.5} & A & 0.006390 & -329.2 & 315.09 & -85.91 \\
\hline & $\mathrm{B}$ & 0.005092 & -314 & 329 & -85.7 \\
\hline \multirow[t]{2}{*}{8.0} & A & 0.004533 & -361.3 & 358.94 & -90.03 \\
\hline & B & 0.003687 & -334 & 373 & -88.4 \\
\hline \multirow[t]{2}{*}{8.5} & A & 0.003248 & -396.1 & 404.93 & -94.24 \\
\hline & B & 0.002722 & -355 & 417 & -90.9 \\
\hline \multirow[t]{2}{*}{9.0} & A & 0.002348 & -434.6 & 453.02 & -98.63 \\
\hline & B & 0.002045 & -376 & 463 & -93.2 \\
\hline \multirow[t]{2}{*}{9.5} & $\mathrm{~A}$ & 0.001707 & -475.4 & 503.37 & -103.03 \\
\hline & $\mathrm{B}$ & 0.001560 & -395 & 510 & $\begin{array}{l}-95.3 \\
\end{array}$ \\
\hline \multirow[t]{2}{*}{10.0} & A & 0.001246 & -516.9 & 556.08 & -107.30 \\
\hline & B & 0.001207 & -412 & 559 & -97.1 \\
\hline
\end{tabular}


V. Table SI-3. Values of the low and high pressures used in the EOS calculation at temperatures 3.0 to $10.0 \mathrm{~K}$ along with the respective low and high mole fractions of dimer and trimer. The corresponding high and low values of the monomer mole fractions are 0.995 and 0.990 , respectively.

\begin{tabular}{|l|l|l|l|l|l|l|}
\hline & \multicolumn{3}{|c|}{$x_{\text {m,high }}=0.995$} & \multicolumn{3}{c|}{$x_{\text {m,low }}=0.990$} \\
\hline$T / \mathrm{K}$ & $P_{\text {low }} /$ bar & $x_{\text {D,low }}$ & $x_{\mathrm{T}, \text { low }}$ & $P_{\text {high }} /$ bar & $x_{\text {D,high }}$ & $x_{\text {T,high }}$ \\
\hline 3.0 & 0.010 & 0.00478 & $4.80 \times 10^{-5}$ & 0.021 & 0.00994 & $2.09 \times 10^{-4}$ \\
\hline 3.5 & 0.015 & 0.00512 & $5.43 \times 10^{-5}$ & 0.029 & 0.00980 & $2.00 \times 10^{-4}$ \\
\hline 4.0 & 0.020 & 0.00507 & $5.26 \times 10^{-5}$ & 0.039 & 0.00979 & $1.97 \times 10^{-4}$ \\
\hline 4.5 & 0.026 & 0.00506 & $5.17 \times 10^{-5}$ & 0.051 & 0.00982 & $1.96 \times 10^{-4}$ \\
\hline 5.0 & 0.033 & 0.00505 & $5.08 \times 10^{-5}$ & 0.065 & 0.00985 & $1.94 \times 10^{-4}$ \\
\hline 5.5 & 0.040 & 0.00491 & $4.74 \times 10^{-5}$ & 0.081 & 0.00985 & $1.92 \times 10^{-4}$ \\
\hline 6.0 & 0.049 & 0.00491 & $4.67 \times 10^{-5}$ & 0.099 & 0.00982 & $1.88 \times 10^{-4}$ \\
\hline 6.5 & 0.060 & 0.00497 & $4.71 \times 10^{-5}$ & 0.12 & 0.00984 & $1.86 \times 10^{-4}$ \\
\hline 7.0 & 0.072 & 0.00498 & $4.67 \times 10^{-5}$ & 0.144 & 0.00987 & $1.84 \times 10^{-4}$ \\
\hline 7.5 & 0.084 & 0.00491 & $4.44 \times 10^{-5}$ & 0.17 & 0.00983 & $1.79 \times 10^{-4}$ \\
\hline 8.0 & 0.10 & 0.00497 & $4.47 \times 10^{-5}$ & 0.20 & 0.00984 & $1.76 \times 10^{-4}$ \\
\hline 8.5 & 0.116 & 0.00494 & $4.31 \times 10^{-5}$ & 0.232 & 0.00978 & $1.70 \times 10^{-4}$ \\
\hline 9.0 & 0.135 & 0.00495 & $4.22 \times 10^{-5}$ & 0.27 & 0.00981 & $1.66 \times 10^{-4}$ \\
\hline 9.5 & 0.156 & 0.00496 & $4.09 \times 10^{-5}$ & 0.312 & 0.00982 & $1.61 \times 10^{-4}$ \\
\hline 10.0 & 0.180 & 0.00498 & $3.98 \times 10^{-5}$ & 0.36 & 0.00986 & $1.57 \times 10^{-4}$ \\
\hline
\end{tabular}


VI. Table SI-4. Values of the LHS of eq 19 of the article between 3.0 and $10.0 \mathrm{~K}$ at the respective low and high pressures used in the statistical thermodynamics calculations.

\begin{tabular}{|l|l|l|l|l|}
\hline$T / \mathrm{K}$ & $\mathrm{P}_{\text {low }} /$ bar & Value $^{\mathrm{a}}$ & $\mathrm{P}_{\text {high }} /$ bar & Value $^{\mathrm{a}}$ \\
\hline 3.0 & 0.010 & 0.000810 & 0.021 & 0.00170 \\
\hline 3.5 & 0.015 & 0.000827 & 0.029 & 0.00160 \\
\hline 4.0 & 0.020 & 0.000789 & 0.039 & 0.00154 \\
\hline 4.5 & 0.026 & 0.000765 & 0.051 & 0.00150 \\
\hline 5.0 & 0.033 & 0.000746 & 0.065 & 0.00147 \\
\hline 5.5 & 0.040 & 0.000712 & 0.081 & 0.00144 \\
\hline 6.0 & 0.049 & 0.000702 & 0.099 & 0.00142 \\
\hline 6.5 & 0.06 & 0.000704 & 0.12 & 0.00141 \\
\hline 7.0 & 0.072 & 0.000702 & 0.144 & 0.00140 \\
\hline 7.5 & 0.084 & 0.000689 & 0.17 & 0.00139 \\
\hline 8.0 & 0.10 & 0.000698 & 0.20 & 0.00140 \\
\hline 8.5 & 0.116 & 0.000696 & 0.232 & 0.00139 \\
\hline 9.0 & 0.135 & 0.000702 & 0.27 & 0.00140 \\
\hline 9.5 & 0.156 & 0.000708 & 0.312 & 0.00142 \\
\hline 10.0 & 0.18 & 0.000719 & 0.36 & 0.00144 \\
\hline
\end{tabular}

a Values of the LHS of eq 19 of the article. 